\title{
Strategie di ridefinizione semantica della città \\ La street art e i segni di riscrittura urbana
}

\section{Orlando Paris}

Università per Stranieri di Siena, Dipartimento di Ateneo per la Didattica e la Ricerca (DADR), IT paris@unistrasi.it

\begin{abstract}
In this paper we will analyse street art by focusing on the effects of meaning that this form of art produces in its relationship to urban elements and spaces and, conversely, how these enter significantly into artistic representation. In other words, the central theme will be the complex relationship between pieces of street art and the surfaces used (walls, shutters, barriers, buildings, etc.), which are anything but neutral locations. This structural relationship - which is an internal mechanism of the urban text - raises broad and specifically semiotic questions such as the representation of a city, its internal conflicts and the subjectivities that seek to re-write it.
\end{abstract}

\section{Key Words}

Semiotics; Street art; Urban text; Surface; Re-writing signs.

\section{Sommario/Contents}

1. La street art e il suo supporto: un dialogo bidirezionale

2. Riscrivere la città: mettere in primo piano o cancellare il supporto

3. Alcuni esempi di riscrittura urbana: muri, barriere ed edifici

Bibliografia 


\section{Ocula ${ }^{\text {Flux_saggi }}$ \\ Occhio semiotico sui media | Semiotic eye on media}

Orlando Paris | Strategie di ridefinizione semantica della città

\section{La street art e il suo supporto: un dialogo bidirezionale}

Muri, barriere, pareti mobili o immobili, saracinesche, portoni e porte, ma anche palazzi in disuso e cartelli stradali sono parte delle geometrie architettoniche delle città, come lo sono strade, piazze, vicoli e quartieri. Spazi ed elementi che sono dotati di senso poiché socialmente determinati. Spazi ed elementi attraversati, iscritti, dai segni lasciati dalle attività antropiche: scritture esposte, manifesti pubblicitari, immagini, graffiti-writing, opere di street art, e così via (cfr. Petrilli 2017). La città è uno spazio stratificato e multiforme in cui ognuno degli elementi sopracitati e ogni segno può essere analizzato e letto autonomamente, ma rimane comunque una parte di un tutto significante che produce senso altresì nella relazione con le altre componenti del tessuto cittadino. In questa prospettiva i panorami semiotici urbani contemporanei sono un complesso coacervo di testi, e quindi di linguaggi differenti, che entrano in connessione l'uno con l'altro. In questo lavoro la street art verrà analizzata proprio da questo punto di vista, concentrandosi sugli effetti di senso che produce nella relazione con gli elementi e gli spazi urbani e, viceversa, su come questi ultimi entrino in maniera significativa nella rappresentazione artistica. È quindi il rapporto complesso tra le opere di street art e i loro supporti (muri, barriere, saracinesche, ecc.) che verrà indagato, supporti tutt'altro che neutri, tele anomale. Focalizzandosi su questa relazione strutturale, che non è altro che un meccanismo interno al testo urbano, ${ }^{1}$ si possono analizzare le importanti dinamiche di senso che si innescano tra l'opera d'arte e il suo supporto, ma non solo, e sarebbe riduttivo fermarsi qui. Questa relazione opera-supporto, infatti, proprio nel contesto urbano porta alla luce temi più ampi e squisitamente semiotici, che riguardano la rappresentazione della città, le dinamiche identitarie che la abitano, le conflittualità sociali che la attraversano e le soggettività che la vogliono ri-scrivere (v. Mastroianni 2013). In questo senso la street art si presenta come un caso di studio esemplare per mettere in luce i meccanismi semiotici che caratterizzano il tessuto urbano e, soprattutto, le dinamiche di riscrittura e rifunzionalizzazione che lo attraversano.

Nelle prossime pagine distingueremo due diverse modalità di riscrittura del testo urbano: le riscritture esplicite (opere di street art che sono dei meta-segni) e le riscritture implicite (opere di street art che sono segni): una distinzione, questa, fondata proprio a partire dalla relazione che si instaura tra opera e supporto. Inoltre, analizzeremo il doppio movimento che si innesca tra queste due entità strutturali dell'opera artistica (opera/supporto): se per un verso i supporti anomali interagiscono in vario modo con le figure della rappresentazione, dall'altro l'opera dialoga con i significati che questi supporti hanno e in alcuni casi arriva a rifunzionalizzarli.

1 Sulla nozione di città come testo si rimanda a: Volli (2005, 2008, 2009); Marrone (2009); Marrone-Pezzini (2006; 2008). 


\section{Ocula ${ }^{\text {Flux__saggi }}$ \\ Occhio semiotico sui media | Semiotic eye on media}

Orlando Paris | Strategie di ridefinizione semantica della città

\section{Riscrivere la città: mettere in primo piano o cancellare il supporto}

"L’immagine mi dice se stessa" - vorrei dire. Cioè, il suo dirmi qualcosa consiste nella sua propria struttura, nelle sue forme e nei suoi colori.

Ludwig Wittgenstein, Ricerche filosofiche, 523

Louis Marin, filosofo francese e tra i fondatori della semiotica dell'arte, riflettendo sulla rappresentazione pittorica nel Quattrocento sottolineava come alla genesi del senso di un'opera contribuissero anche quei dispositivi che lui definiva "presentativi: l'inquadratura e i suoi margini, le cornici interne ed esterne al campo figurativo, la scenografia di un dipinto e tutto il lavoro della pittura tra piano, superficie, sfondo e supporto della rappresentazione» (Marin 2012: 95). In altre parole, tutta un'impalcatura dell'opera d'arte che «la tiene e la espone, dissimulandosi tuttavia al punto da passare inosservata» (Marin 2012: 7). Per capire il ruolo di questi dispositivi presentativi, fondamentali nell'analisi che ci apprestiamo a fare della street art, bisogna però fare un piccolo passo indietro e soffermarci sulla nozione di rappresentazione (e di segno) così come intesa da Louis Marin. Una nozione fondata sul concetto di sostituzione e, quindi, fortemente debitrice alla teoria del segno dei logici di Port-Royal: "rappresentare" significa, prima di tutto, "presentare qualcuno o qualcosa di assente, renderlo presente attraverso un segno" (Marin 2012: 7). Per Marin, però, ogni "rappresentazione" nel momento in cui "presenta" qualcosa o qualcuno di assente mostra anche sé stessa, a volte esibendo e a volte occultando la sua presenza (cfr. Scalabroni 2008). Esiste, in altre parole, una dimensione transitiva del segno (rappresentare qualcosa) e una dimensione riflessiva (presentarsi): questa seconda componente si potrebbe definire metarappresentativa e sposta l'attenzione sulla natura segnica della rappresentazione visiva, sui meccanismi che la caratterizzano. Proprio di questa dimensione riflessiva fanno parte quei congegni che vengono definiti di presentazione e con cui abbiamo aperto questo paragrafo. Congegni attraverso cui la "rappresentazione" mostra le sue caratteristiche funzionali, la sua struttura di segno. Luisa Scalabroni (2008), in un'analisi approfondita degli studi di Marin, ripercorre alcuni di questi concetti chiave tra cui quello di "supporto" e "sfondo" che sono centrali nella nostra analisi. Lo "sfondo", supporto materiale e superfice di rappresentazione, è uno di quei dispositivi che Marin definisce di presentazione e sul quale ci concentreremo nelle prossime pagine al fine di comprendere gli effetti di senso che lo riguardano in relazione all'opera di street art:

Lo sfondo, "supporto materiale e superficie d'iscrizione e di figurazione attraverso cui ogni figura si manifesta allo sguardo" è un dispositivo necessario alla presentazione della rappresentazione, che viene denegato nel caso di immagini che ricorrono all'uso della prospettiva lineare al fine di creare l'illusione di una profondità o, al contrario, valorizzato nella sua bidimensionalità quando, negando la profondità e la sua relazione con le figure, ne viene manifestato il suo essere superficie. Lo sfondo emerge, in questo 
Orlando Paris | Strategie di ridefinizione semantica della città

caso, verso lo spettatore e "ci appare come superficie, ed è a questo punto che il quadro si presenta come quadro, che si presenta cioè, non come rappresentante qualcosa quanto come rappresentazione". (Scalabroni 2008: 8)

Il "supporto" può essere negato, cancellato, attraverso diverse tecniche tra le quali l'uso della prospettiva e, quindi, attraverso la costruzione dell'illusione della profondità. La macchina prospettica, ci dice Marin, nega lo sfondo e nega il supporto, lo penetra per spingersi in lontananza, o addirittura, fino all'infinito, «un infinito che è rappresentato in pittura dal trattamento atmosferico dell'orizzonte, un limite che si apre all'illimitato» (Marin 2001: 198). Emerge, in questo modo, tutta la forza della dimensione transitiva del segno, e cioè viene esaltata la "cosa" rappresentata in chiave artistica: l'opera, oggetto di contemplazione, è fondata sull'illusione mimetica. Alcune volte, al contrario, il "supporto" emergere verso lo spettatore, viene portato in primo piano, ci appare come superficie e condizione necessaria della rappresentazione, ed è a questo punto che per Marin il quadro si presenta anche come quadro, non solo come rappresentante qualcosa ma come rappresentazione (cfr. Marin 2001): emerge, quindi, una dimensione riflessiva del segno, il quale si mostra anche in quanto segno. Proprio la street art oltre a rendere più chiaro il meccanismo descritto da Marin, potrebbe rappresentare il banco di prova per l'avvio di una riflessione su alcuni di questi dispositivi presentativi, in particolare sul "supporto".

Marin si concentra sulla relazione "supporto"/"opera" in diverse occasioni, in modo significativo nell'analisi degli affreschi di Filippo Lippi a Prato e in particolare nell'analisi dell'episodio dell'esecuzione di Giovanni in cui il boia tende la testa mozzata verso il vassoio di Salomè. In questo affresco, in una parete è rappresentato il corpo del Santo con il boia, mentre il braccio di quest'ultimo che solleva la testa di Giovanni - per darla a Salomè - passa su un'altra parete e il taglio dell'angolo del muro coincide esattamente con il gomito dell'esecutore. Un'articolazione del supporto (il limite del muro che si piega ad angolo retto) coopera con le figure della rappresentazione:

la decapitazione di Giovanni [...] riceve tutta la violenza della separazione mortale dall'articolazione dei due muri [...]. Sorpresa dell'occhio, choc dello sguardo contemplatore, lui stesso scisso su questo angolo dalla trasgressione della cornice architettonica della rappresentazione. (Marin 2012: 213)

Nella street art il rapporto "opera"/“supporto" diventa molto più intenso e complesso rispetto all'arte del Quattrocento analizzata da Marin: prima di tutto poiché in questa pratica artistica i supporti sono elementi urbani (muri, cartelli stradali, porte, portoni, saracinesche, vagoni dei treni, palazzi, eccetera...) dotati di una propria funzione e di un proprio significato con cui l'opera è costretta a fare i conti. Per questa ragione il rapporto strutturale "opera"/“supporto", a nostro avviso, diventa decisivo per capire le ragioni semiotiche che portano a leggere la street art come una dinamica di riscrittura urbana e comprendere le modalità con cui questa riscrittura si attua. Sono due le tendenze che abbiamo rintracciato: ci sono casi, la maggior parte, in cui il supporto viene valorizzato, viene reso visibile e coopera esplicitamente con le figure 


\section{Ocula ${ }^{\text {Flux_saggi }}$ \\ Occhio semiotico sui media | Semiotic eye on media}

Orlando Paris | Strategie di ridefinizione semantica della città

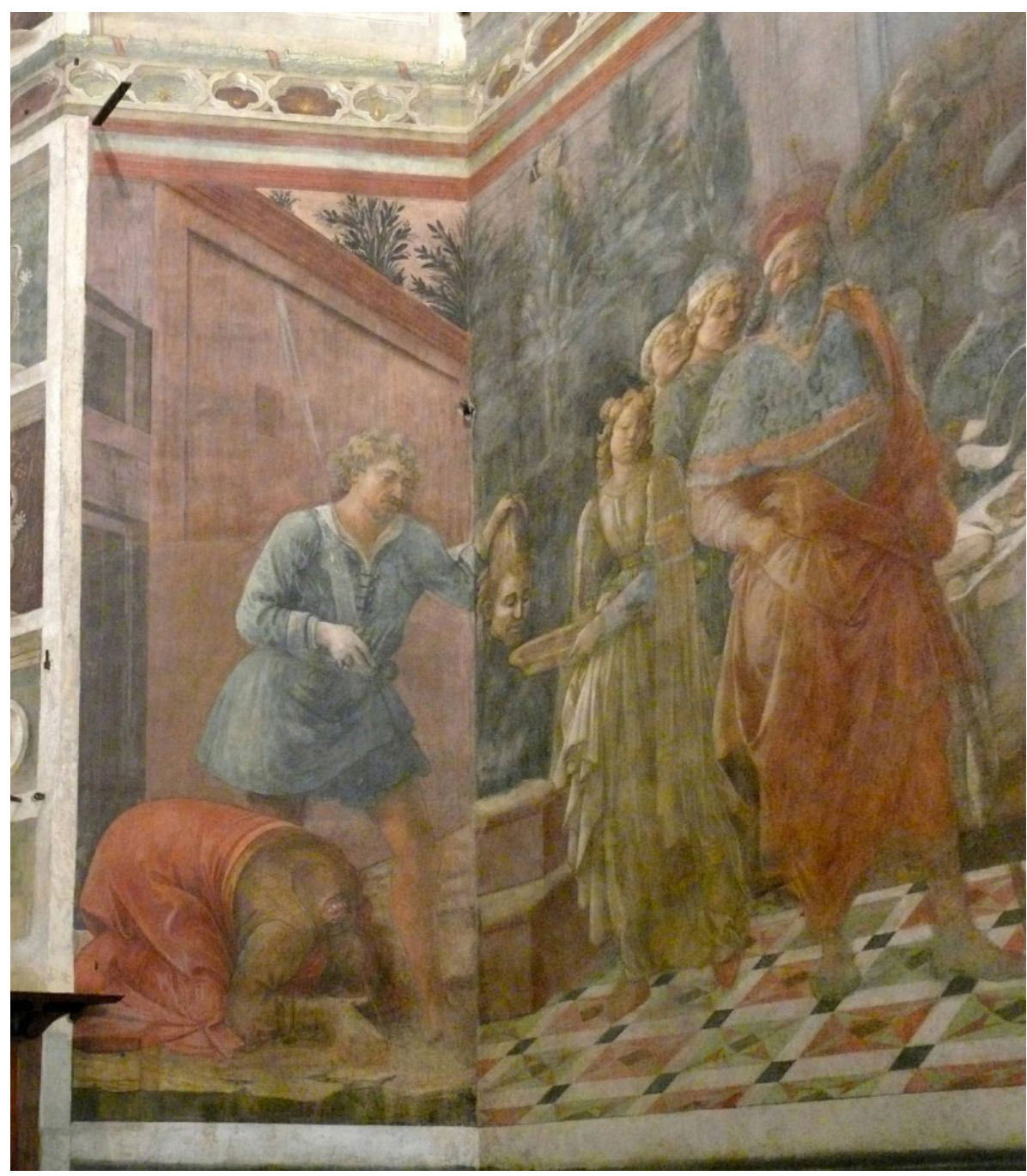

Figura 1. Filippo Lippi, Decollazione di san Giovanni Battista, Duomo, Prato.

della rappresentazione (prevale una dimensione riflessiva del segno); mentre altri casi in cui il supporto viene occultato o negato (prevale una dimensione transitiva del segno). Ad ognuna di queste due modalità, come vedremo nelle prossime pagine, corrisponde una specifica dinamica di "riscrittura" urbana: nel primo caso ci troviamo di fronte ad una "riscrittura esplicita" (meta-segni che si presentano come segni di ri-scrittura); nel secondo caso ci troviamo di fronte ad una "riscrittura implicita" (segni che rappresentano qualcosa e solo indirettamente riscrivono il testo urbano).

2.1 I meta-segni e la "riscrittura esplicita" della città: mettere in primo piano il supporto

In molti dei casi che abbiamo analizzato gli street artist sembrano fondare le loro opere sulla valorizzazione del "supporto", sulla sua trasformazione in "figura della rappresentazione": proprio questa dinamica interna all'opera, in 


\section{Dcula \\ Occhio semiotico sui media | Semiotic eye on media}

Orlando Paris | Strategie di ridefinizione semantica della città

queste occasioni, è ciò che ci porta a leggere la street art come una pratica di riscrittura urbana. Il supporto non viene più cancellato o negato attraverso l'utilizzo della macchina prospettica, ma viene fatto emergere, riportato in primo piano e così facendo, come vedremo negli esempi che seguono, viene dotato di nuovo significato. Questa dinamica - far emergere il supporto o semplicemente valorizzarlo rendendolo esplicito - diventa l'emblema di una rappresentazione artistica che decide di giocare su un piano diverso da quello della sola operazione mimetica, diverso da quello dell'opera davanti alla quale fermarsi in contemplazione poiché ci rappresenta artisticamente la realtà fenomenologica: paesaggi ideali, pastorali, eroici; o anche ritratti divini, storie religiose e così via. L'emersione del supporto è emblema di una rappresentazione fondata invece sulla sua dimensione riflessiva, metalinguistica, che porta in primo piano la natura segnica e il meccanismo strutturale che la caratterizza: un meccanismo che, in questo caso, potremmo definire di ri-scrittura, una riscrittura esplicita poiché esplicitata nell'opera stessa. Non una "mimesis"

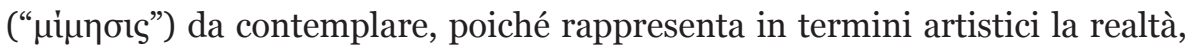
ma un'operazione meta-rappresentativa: l'opera ci parla del suo supporto e diventa prima di tutto un segno di riscrittura. A tale proposito possiamo citare Clet Abraham, street artist francese che lavora in Italia dal 1990. Le sue opere sono realizzate attraverso l'applicazione di adesivi alla segnaletica stradale e sono fondate proprio sul rapporto "opera"/"supporto", sulla trasformazione di quest'ultimo in "figura della rappresentazione".

Nella figura 2 possiamo vedere una delle opere di Abraham: la freccia di un segnale stradale che indica l'obbligo di svoltare a destra diventa il naso di un Pinocchio disegnato dall'artista. Il supporto è il cartello stradale che non viene coperto o negato per costruire l'illusione mimetica dell'opera da contemplare;

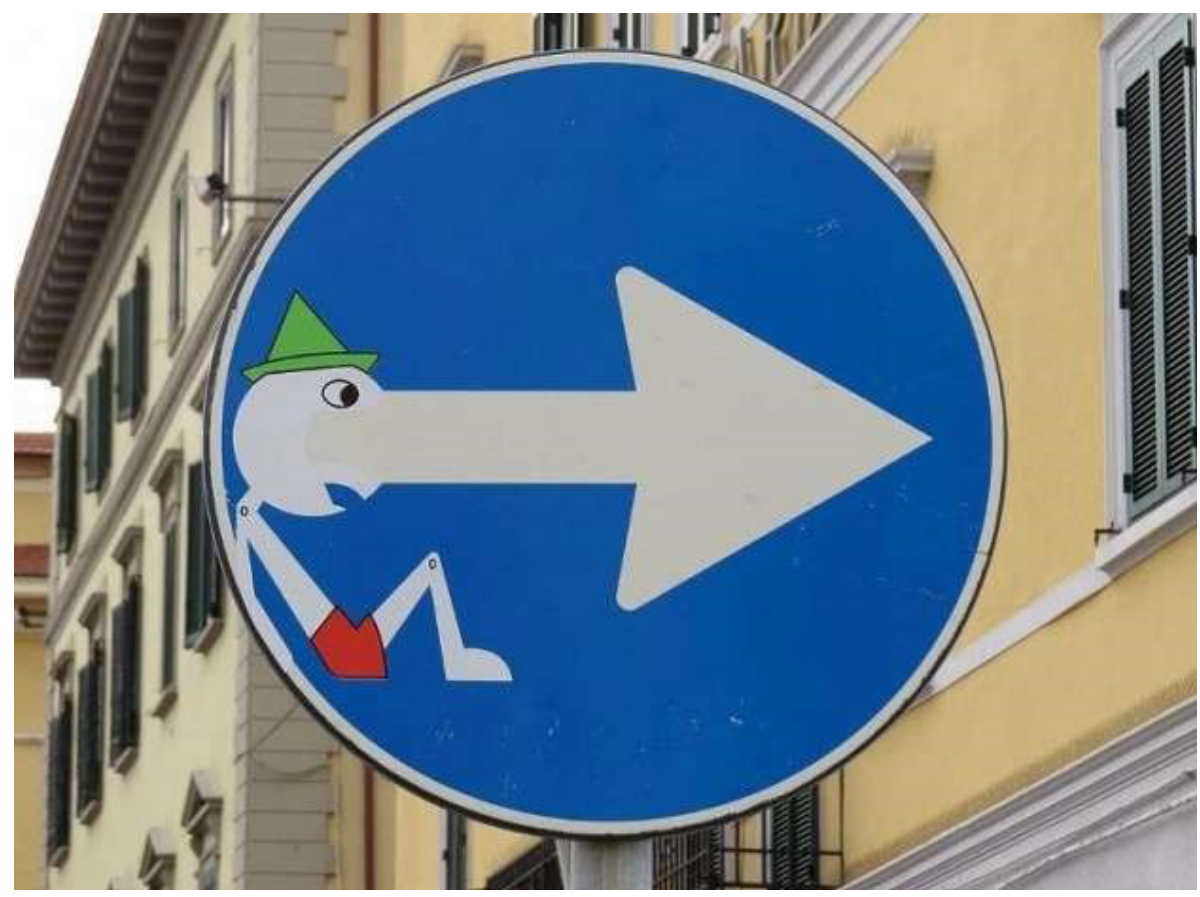

Figura 2. Clet Abraham, Firenze 


\section{Dcula \\ Occhio semiotico sui media | Semiotic eye on media}

Orlando Paris | Strategie di ridefinizione semantica della città

al contrario, oltre a rimanere visibile coopera con le figure della rappresentazione e diviene esso stesso una figura della rappresentazione: il naso di Pinocchio appunto. Ma la freccia rimane visibile e interpretabile anche nel suo significato originario ("obbligo di svoltare a destra"), l'osservatore di fronte all'opera è quindi consapevole di questa doppia dinamica di significazione e di una doppia possibilità di interpretazione: il significato originario, "svoltare a destra", e la riscrittura artistica, "il naso di Pinocchio". Il segno artistico, in questo modo, esplicita il meccanismo che lo caratterizza: prima di rappresentare qualcosa (funzione transitiva), questo rende esplicito e visibile il suo congegno "meta-rappresentativo" (funzione riflessiva) e diventa, in altre parole, un segno di una ri-scrittura, un meta-segno che mostra la trasformazione di un enunciato urbano che altro non è che il proprio supporto. Meta-segno, quindi, poiché ci parla della sua dinamica di funzionamento e ce la mostra: questa tipologia di opere si liberano della necessità di essere repliche della realtà fenomenologica e diventano, prima di tutto, delle "prese di parola", delle riscritture esplicite del testo urbano. Stesso meccanismo è visibile nella figura 3 , il segnale stradale diventa una figura della rappresentazione e l'opera contribuisce alla sua rinegoziazione, ridefinisce il suo significato originario, in questo caso negando il divieto. Ci troviamo, di nuovo, davanti ad un meta-segno che ci esplicita e ci mostra la riscrittura del suo supporto.

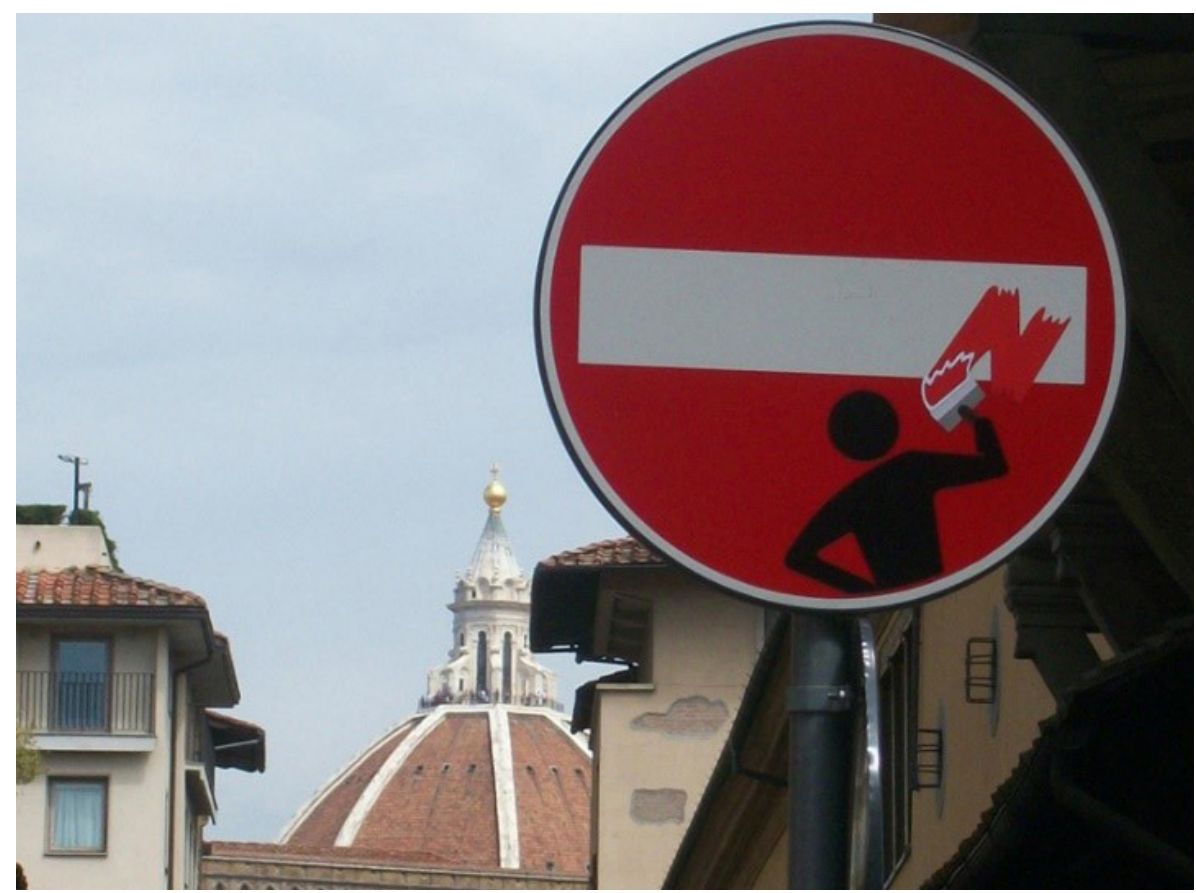

Figura 3. Clet Abraham, Firenze

Un altro interessante artista che ci può aiutare a comprendere meglio la dinamica che stiamo descrivendo è Exit Enter, attivo in Italia fin dal 2013. In queste immagini (figg. 4-5) si può vedere come il muro, con le sue caratteristiche di superficie (fig. 5), ma anche con gli artefatti che lo abitano (fig. 4), diven- 


\section{Dcula $a^{\text {P1ux_saggi }}$ \\ Occhio semiotico sui media | Semiotic eye on media}

Orlando Paris | Strategie di ridefinizione semantica della città

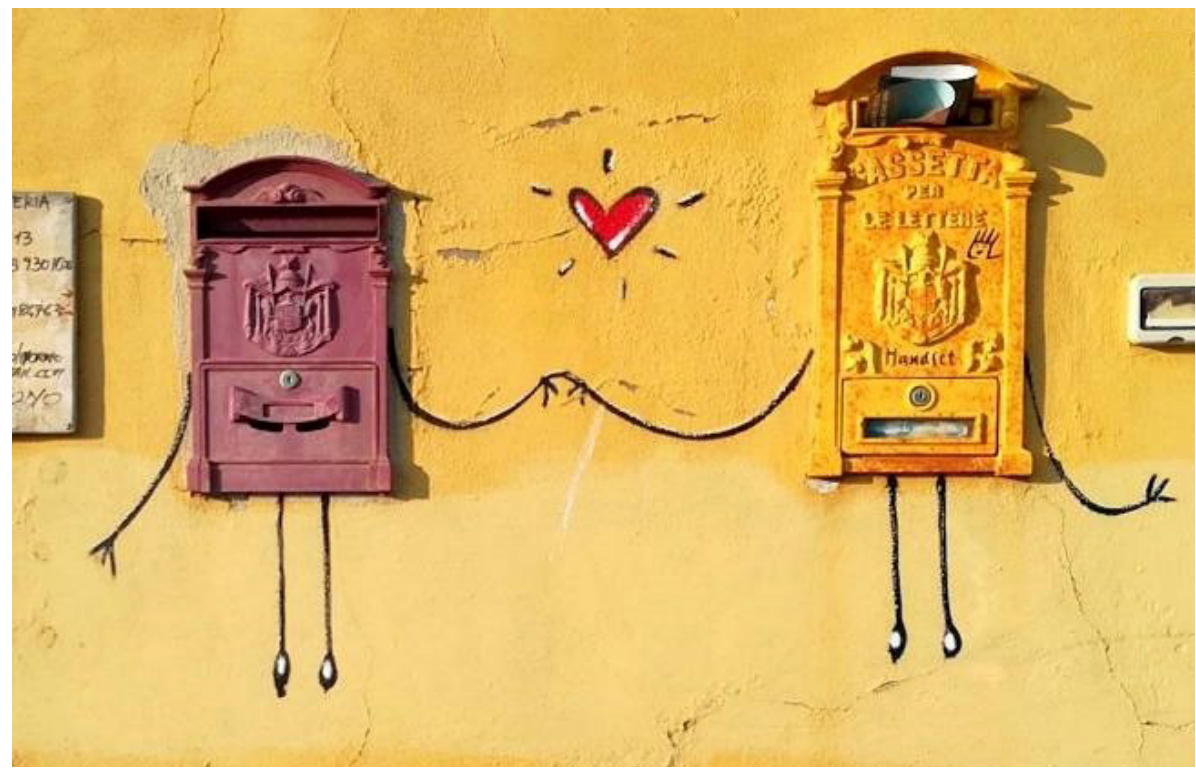

Figura 4. Exit Enter, Pisa

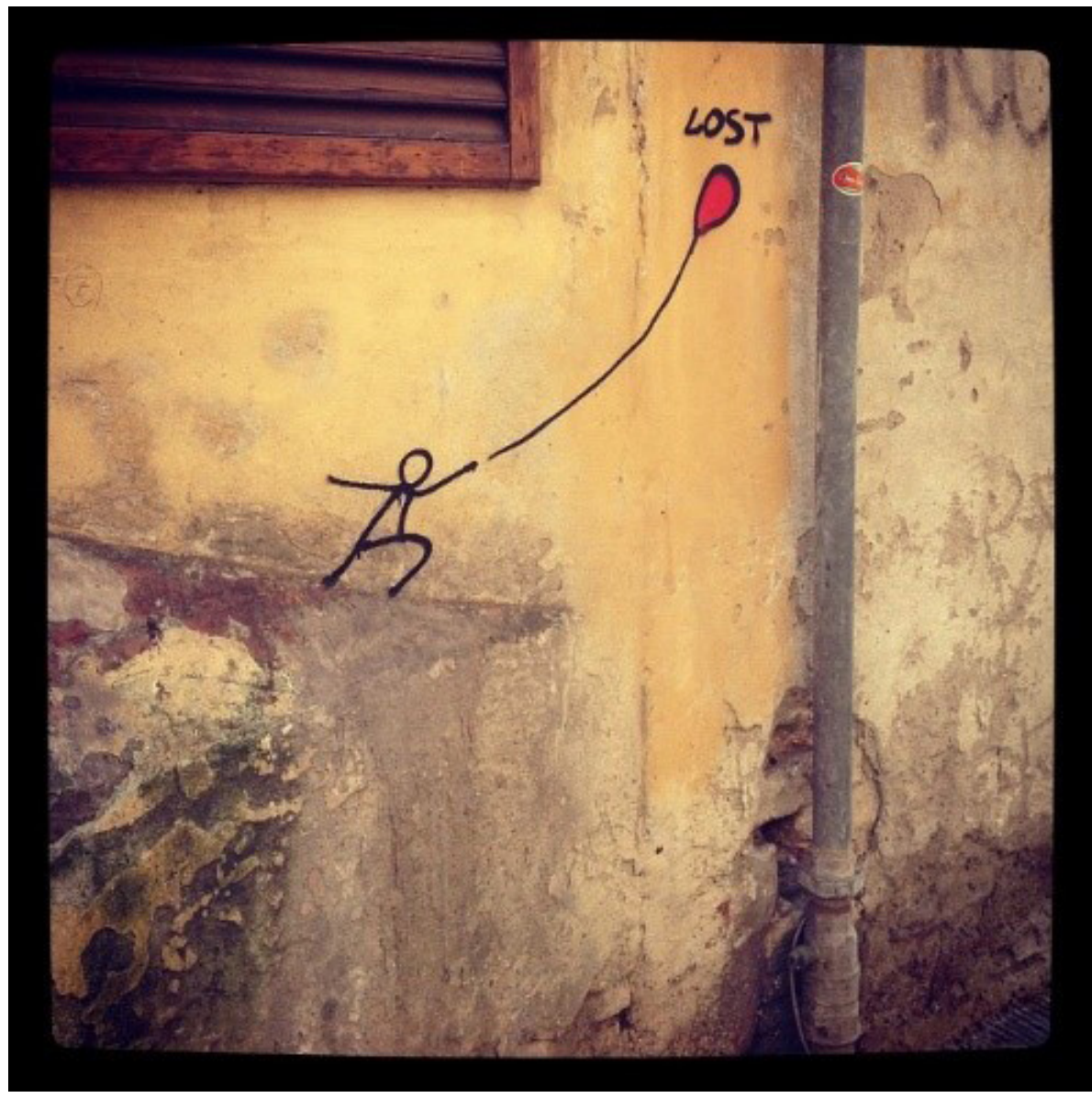

Figura 5. Exit Enter, Firenze 


\section{Dcula \\ Flux_Saggi \\ Occhio semiotico sui media | Semiotic eye on media}

Orlando Paris | Strategie di ridefinizione semantica della città

ti componente costitutiva dell'opera stessa: il supporto coopera con le figure della rappresentazione e diventa esso stesso una figura della rappresentazione attivando un meccanismo che è quello descritto per la figura 2 e per la figura 3 .

Infine, solo per citare un autore di cui ci occuperemo nel prossimo paragrafo, è un segno di riscrittura esplicita l'opera di Banksy realizzata sul muro costruito in Cisgiordania. Lo street artist disegna due angioletti che tentano di scardinare la barriera di separazione (fig.6), la quale in questo modo, oltre ad essere il supporto dell'opera, diventa uno dei soggetti protagonisti della rappresentazione. Il meta-segno è in questo caso ancora più evidente: la rappresentazione ci parla del suo supporto e così facendo lo ri-scrive: una "riscrittura esplicita" che, come vedremo, ha un forte valore politico.

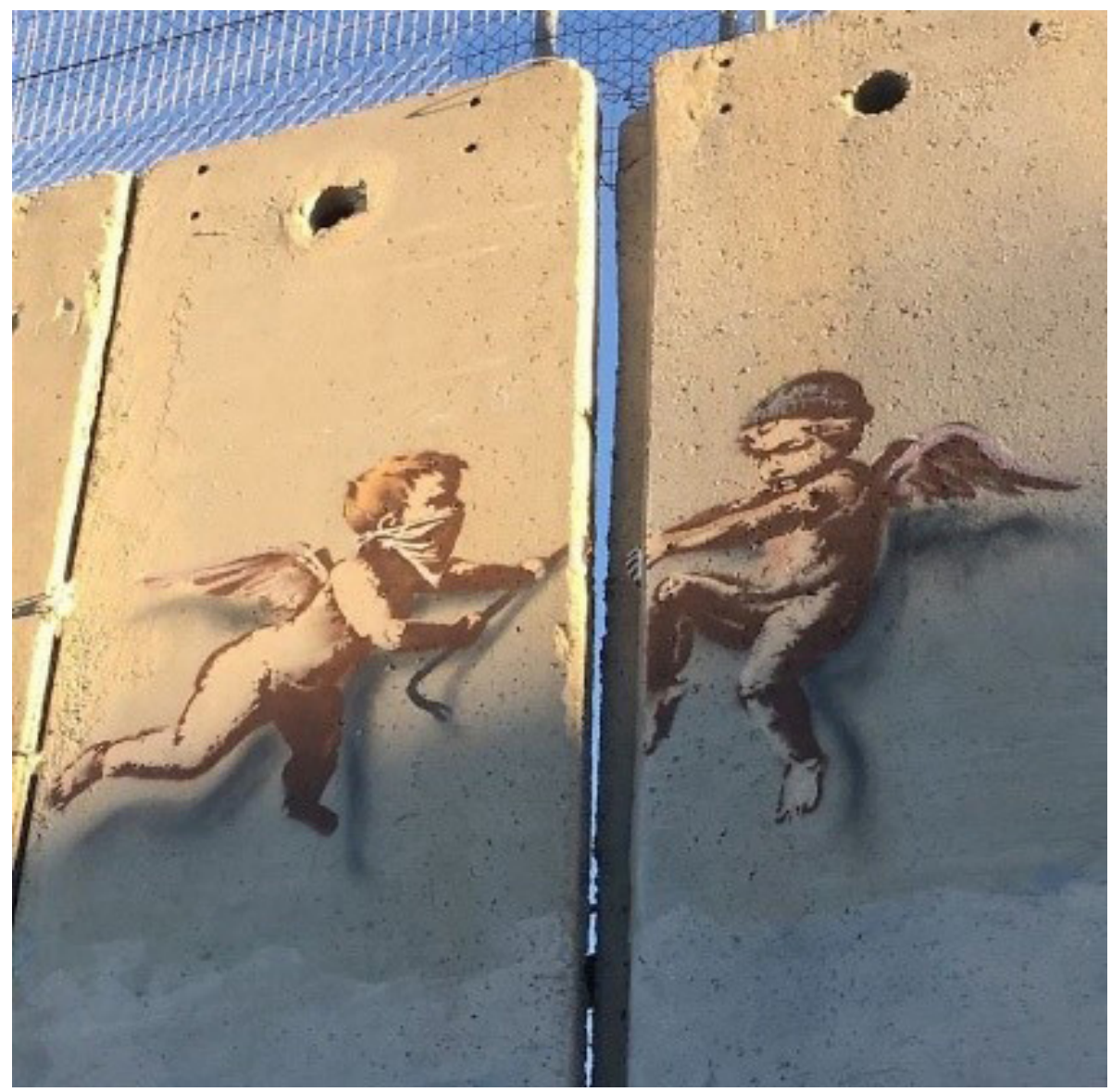

Figura 6. Banksy, Cisgiordania

\subsection{Segni di "riscrittura implicita" urbana: occultare il supporto}

Come abbiamo visto, nella maggior parte dei casi la street art non può fare a meno di rendere conto esplicitamente del suo supporto, di valorizzarlo, problematizzarlo, farlo emergere nell'opera con il fine di risemantizzarlo e riscriverlo. È una sua peculiarità che, come abbiamo visto nelle pagine precedenti, ci ha 


\section{Ocula \\ Flux_Saggi \\ Occhio semiotico sui media | Semiotic eye on media}

Orlando Paris | Strategie di ridefinizione semantica della città

portato a parlare di meta-segni e ci porta a leggere la street art come una pratica di riscrittura urbana. Tuttavia, ci sono anche opere che si fondano su una dimensione transitiva, in cui viene esaltata la "cosa" rappresentata in chiave artistica e dove il supporto viene negato per garantire l'illusione mimetica. Ci troviamo sempre di fronte ad una pratica di ri-scrittura, ma una "riscrittura implicita" diversa da quella analizzata nel punto precedente. Prendiamo come esempio le opere di un affermato street artist australiano, Fintan Magee, caratterizzate da uno stile pittorico-figurativo e dalle dimensioni enormi.

L'opera rappresentata nella figura 7 , intitolata The Rebuild, non è un meta-segno che mostra la riscrittura di un enunciato urbano, ma prima di tutto un segno che ci mostra qualcosa in chiave artistica: una giovane donna che tiene in mano dei rami in un ambiente che sembra essere stato colpito da un'i-

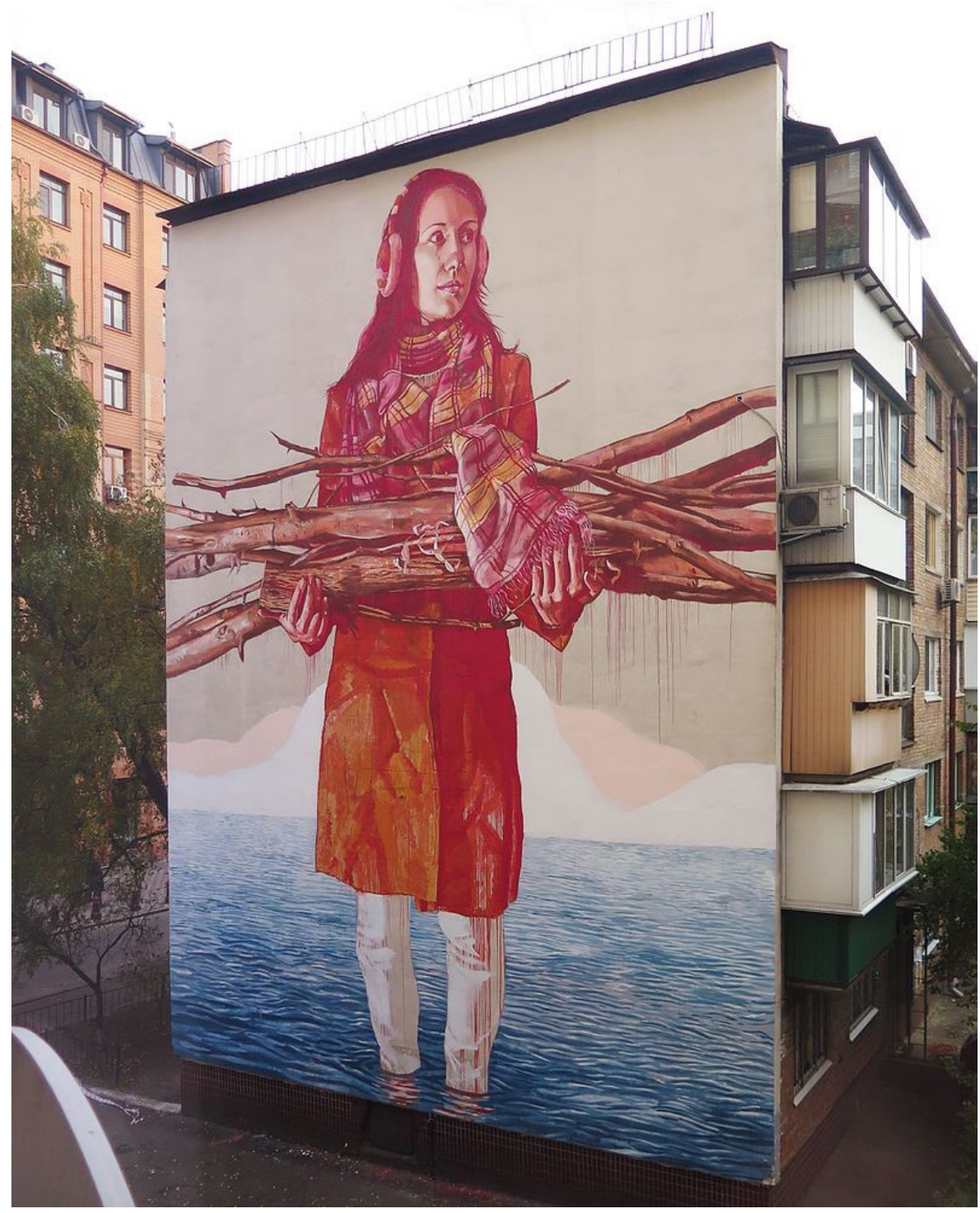

Figura 7. Fintan Magee, Kiev 
Orlando Paris | Strategie di ridefinizione semantica della città

nondazione. Un tema questo che è ricorrente nei lavori di Magee, che ha dedicato un'intera serie delle sue opere alle devastanti alluvioni che nel 2011 hanno colpito la città australiana in cui l'artista è cresciuto, Brisbane. Nelle sue opere le conseguenze delle inondazioni vengono affrontate artisticamente sia in chiave personale, come parte dell'esperienza dell'artista, ma anche in chiave generale focalizzandosi sul rapporto tra uomo e ambiente. In questo caso prevale la dimensione transitiva del segno, ci troviamo di fronte a un'opera che prima di tutto ci rappresenta qualcosa e che dissimula tutto l'apparato presentativo, compreso il supporto. Il supporto scelto da Magee è il muro che non viene fatto emergere, ma al contrario viene occultato attraverso la rappresentazione dello sfondo e attraverso l'utilizzo della prospettiva. Ma questo artefatto, il muro appunto, viene comunque ri-scritto e in qualche modo rifunzionalizzato da quest'opera, anche se non è esplicitamente richiamato nella rappresentazione stessa: si tratta di una ri-scrittura implicita. In questo senso ci sembrano molto interessanti i lavori di Simona Stano (2013) sui graffiti e le pratiche di riscrittura murale, dove la studiosa sottolinea come i muri siano costruiti per ottenere determinati obiettivi, in particolare le loro finalità sembrano associarli a concetti quali il limite, l'isolamento, la sicurezza, l'incomunicabilità eccetera. In altre parole, sono elementi che separano uno spazio precedentemente unitario, continuo, e costruiscono delle opposizioni semantiche molto forti: dentro/fuori, visibile/invisibile, continuo/discontinuo (cfr. Stano 2013: 146). La street art - sottolinea ancora Simona Stano - grazie alle linee, ai contrasti cromatici e alle forme, introduce una variazione nella parete monocromatica del muro intervenendo sugli originali nuclei semantici di cui il muro è portatore: ad "ostacolare lo sguardo" si sostituisce "attrarre lo sguardo", alla "normalizzazione" del muro grigio si sostituisce la "visibilità" dell'opera che lo ricopre, all'artefatto capace di "ostacolare la comunicazione" si sostituisce il "mezzo di comunicazione" (cfr. Stano 2013: 159). In questo senso si può parlare di "riscrittura implicita", il muro cessa di essere solamente strumento impenetrabile di isolamento, di incomunicabilità, di normalizzazione e diventa nuovamente visibile, un vero e proprio mezzo di comunicazione: « [...] la barriera svanisce divenendo uno spazio di espressione per l'artista. Dove prima la gente indicava la parete, ora segnala l'opera; ciò che prima si cercava di evitare, ora diviene fulcro d'interesse» (Ferrara, Mondino e Stano 2012).

\section{Alcuni esempi di riscrittura urbana: muri, barriere ed edifici}

Nelle prossime pagine analizzeremo le dinamiche di riscrittura della street art in due casi "limite": nei muri di separazione di natura geopolitica e negli edifici che possiamo definire contesi, abbandonati o occupati (che vivono una fase di transizione e non hanno una funzione definita nel tessuto urbano). Li definiamo "limite" poiché in questi casi la ri-scrittura della street art e le strategie politiche ufficiali sulla città si contrappongono in modo più intenso e radicale. Diventa quindi esplicito quel fenomeno descritto da Roberto Mastroianni (2013), secondo cui la street culture, di cui la street art è parte, è un insieme di pratiche di «Ri-scrittura urbana che sfidano il senso "funzionalista" delle metropoli, inserendosi in quel "brusio semiotico" che attraversa le città 


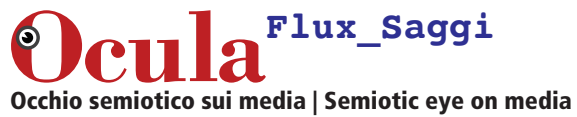

Orlando Paris | Strategie di ridefinizione semantica della città

contemporanee con chiaro intento di mettere in atto pratiche che sfidino le narrazioni dominanti [...]» ( Mastroianni 2013: 48).

Ci focalizzeremo, a tale proposito, su due case study concreti: le "riscritture esplicite" presenti sulla barriera di separazione tra Israele e Palestina in Cisgiordania e la "riscrittura implicita" che ha rifunzionalizzato un fabbricato nella città di Roma (l'ex fabbrica Fiorucci ora Museo dell'Altro e dell'Altrove).

\subsection{Le barriere di separazione geopolitica e la street art: ridefinizione semantica del supporto murario}

Iniziamo sempre dal supporto, dai muri. Cerchiamo di addentrarci all'interno dei significati di cui sono portatori e delle funzioni a cui vengono associati. Secondo Mubi Brighenti:

i muri sono artefatti materiali progettati per promuovere date finalità. Nella maggior parte dei casi si tratta di finalità spaziali, come il recintamento e la separazione. In termini foucaultiani, i muri possono essere descritti come oggetti governamentali. Ciò significa che essi fanno parte di quella attività più ampia che è il governo della popolazione e, come si espresse Foucault (1978/1991: 95), 'il governo non è una questione di imporre delle leggi agli uomini, ma piuttosto di disporre delle cose'. La governamentalità funziona definendo delle posizioni in un campo relazionale di potere, che è essenzialmente di tipo territoriale. La governamentalità adotta perciò un punto di vista strategico sui processi sociali. La strategia mira a controllare lo spazio in base a un piano, e i muri sono pianificati e costruiti come parte di una vera a propria strategia territoriale. Dal punto di vista strategico governamentale i muri funzionano per lo più come separatori, introducendo dei confini in uno spazio precedentemente liscio e permettendo in questo modo la demarcazione di un dentro e di un fuori. (Brighenti 2008: 8)

Parole molto interessanti che sottolineano come i muri siano costruiti con determinate funzioni strategiche, come il recintamento e la separazione, e contemporaneamente siano parte di una vera e propria strategia territoriale.

Compared to the medieval walled city, the modern city transforms walls into elements of a spatial political economy of government. The outer boundary and its capacity to protect the city from external invasion is no longer what really matters (siege being the definitional event of this historic configuration), instead it is the capacity to manage enclaves within the city. As said above, walls become tools for the government of the population. Consequently, it is the power to control settlements and fluxes of people in the urban space that becomes essential. Housing and logistics (stockage, transport, distribution, and delivery) become prominent goals for planners from both economic and political perspectives. (Brighenti 2009: 67)

Brighenti continua in questa analisi sottolineando come pur essendo introdotti come artefatti strategici, i muri sono sempre soggetti a usi tattici attraverso l'interazione di pratiche di resistenza che ne ridefiniscono le funzioni e i significati, tra questi usi tattici c'è proprio la street art. A tale proposito, abbiamo visto nel paragrafo precedente le modalità con cui questa pratica ar- 
Orlando Paris | Strategie di ridefinizione semantica della città

tistica ri-scrive i propri supporti, distinguendo tra una "riscrittura esplicita", in cui il supporto viene esplicitamente richiamato nell'opera, e una "riscrittura implicita", in cui il supporto viene occultato ma comunque riscritto e rifunzionalizzato. Ma non tutti i muri sono uguali, ce ne sono alcuni semanticamente più carichi, come ad esempio le barriere artificiali geopolitiche: muri che dividono popoli, culture, società e che funzionano come elementi fortemente separatori nei quali, quelle opposizioni semantiche che abbiamo descritto precedentemente diventano più forti e radicali (dentro/fuori, visibile/invisibile, continuo/discontinuo, ma anche libertà/prigionia) (cfr. Stano, 2013). Muri, le barriere artificiali geopolitiche, che oltre ai significati "denotativi” richiamano dei significati "connotativi”, dei significati culturali: a questo livello è importante considerare anche l'insieme delle rappresentazioni che ognuno di questi artefatti assume all'interno dei testi complessi di una cultura, l'insieme dei discorsi che lo riguardano e con cui, in qualche modo, viene costruito anche come oggetto culturale. Prendiamo come esempio il "Muro di Berlino", che aveva una funzione di separazione molto forte (politica, geografica, sociale, culturale) e richiamava tutta una serie di radicali opposizioni semantiche, ma era (e lo è ancora oggi) anche il simbolo di un determinato momento storico, della Guerra Fredda, la Cortina di Ferro che divideva il mondo in due blocchi, simbolo del controllo e della violenza politica e così via. Le dinamiche di ri-scrittura di questo artefatto, come vedremo, non possono fare a meno di confrontarsi con entrambi i livelli di significato (denotativo e connotativo). Nel 1989, dopo il crollo del muro, artisti da tutto il mondo intervennero con le loro opere sul lato est della barriera facendo così nascere la Est Side Gallery: una parte del muro lunga circa 1300 metri con un centinaio di opere di street art, una vera e propria galleria a cielo aperto. Proprio su questi interventi artistici si concentra il già citato lavoro di Simona Stano (Stano, 2013), la quale riflette sul processo di ridefinizione dello spazio pubblico di cui abbiamo parlato nelle pagine precedenti e, quindi, sul perché è possibile parlare di street art come pratica di riscrittura murale. Grazie all'intervento artistico, sottolinea la Stano, «il muro cessa dunque di essere uno strumento impenetrabile di isolamento e incomunicabilità e si converte in un vero e proprio mezzo di comunicazione che offre a chiunque la possibilità di esprimersi, e in definitiva, di percepire sé stesso come soggetto attivo» (Stano 2013: 159). Inoltre, al di là di queste riconversioni prettamente funzionali, la Stano sottolinea come si attivi un vero e proprio processo di ri-semantizzazione: le opere rappresentate sul Muro di Berlino non possono far a meno di confrontarsi con i significati "connotativi" di cui quella barriera è portatrice per contrapporvi i contenuti dell'opera. Il Muro di Berlino, in questo modo, cessa di essere solamente il simbolo della Guerra Fredda, del mondo diviso in blocchi, del controllo politico, ma attraverso le opere d'arte - come Dancing to freedom di Jolly Kunjappu, La Buerlinca di Stephan Cacciatore, The mortal kiss di Dimitrij Vrue e le tante altre che ricoprono i 1300 metri della East Side Gallery - diventa anche «un richiamo alla fraternità ritrovata, un inno alla cooperazione e alla convivenza pacifica» (Stano 2013: 160). Le opere citate, utilizzando le categorie che abbiamo delineato nel precedente paragrafo, sono dei "segni" di "riscrittura implicita" della barriera: si mostrano prima di tutto come opere d'arte che 
Orlando Paris | Strategie di ridefinizione semantica della città

rappresentano qualcosa e non come "meta-segni" di "riscrittura", non c'è alcun richiamo esplicito del supporto all'interno delle rappresentazioni artistiche. Ma anche in questo caso, la street art, oltre a rifunzionalizzare la barriera, a trasformarla in una galleria d'arte a cielo aperto, come abbiamo visto attua anche una riscrittura sul livello dei significati "connotativi".

Questo particolare caso che ci descrive Simona Stano, vede gli street artist intervenire su una barriera già abbattuta e che quindi ha perso la sua funzione principale. Cosa succede quando la barriera è ancora in piedi? Quando è ancora attiva?

\subsection{Il Muro in Cisgiordania: abbattere o superare la barriera}

La barriera di separazione costruita da Israele in Cisgiordania nel 2002 è lunga circa $730 \mathrm{~km}$ e formalmente ha lo scopo di impedire l'intrusione di "terroristi palestinesi" nel territorio israeliano. È stata chiamata in diversi modi, Muro della vergogna, Muro dell'annessione, Muro dell'apartheid o anche Muro di separazione razziale. Eyal Weizman nel suo testo Architettura dell'occupazione (Weizman 2009) la definisce una frontiera coloniale mobile. Questi diversi appellativi con cui la barriera è identificata sono la dimostrazione del suo forte valore simbolico: simbolo di divisione, di separazione, incomunicabilità, ma soprattutto simbolo di sopraffazione per la popolazione palestinese che vive in quel territorio. Anche in questo caso sono centinaia gli street artist intervenuti sulla barriera, tanto da avviare quel processo di rifunzionalizzazione descritto per il Muro di Berlino e, quindi, farla diventare una sorta di galleria a cielo aperto: in questo caso sono organizzate delle vere e proprie visite guidate ufficiali nei settori del muro in cui sono presenti le opere degli artisti più famosi, come Banksy. Ma molte delle opere presenti su questa barriera di separazione fanno qualcosa in più, anche in questo caso entrano in connessione con i significati "connotativi" che il supporto richiama, avviando un vero e proprio confronto politico: opere che portano avanti dei discorsi molto chiari, che in modi diversi, evocano il superamento della barriera stessa, la sua distruzione, il suo abbattimento. Evocano, in altre parole, l'abbattimento del supporto su cui sono realizzate. La maggior parte delle opere che analizzeremo sono dei meta-segni di "riscrittura esplicita": opere artistiche che si presentano prima di tutto come ri-scritture, dove il supporto viene chiamato in causa direttamente all'interno della rappresentazione e, in molti casi, ne diventa il soggetto protagonista. Ma, allo stesso tempo, sono anche dei segni che ci mostrano dei significati in aperta opposizione con le connotazioni della barriera. A differenza della Est Side Gallery (dove abbiamo visto la funzione principale della barriera era già venuta meno e la street art interviene dopo il crollo del Muro con rappresentazioni che sono segni di "riscrittura implicita") in questo caso la barriera viene chiamata in causa direttamente nelle rappresentazioni: la "presa di parola" è più forte, la ri-scrittura diventa più radicale, lo scontro tra i contenuti dell'opera e gli originali contenuti della barriera si fa più aspro.

Iniziamo la nostra analisi con uno degli artisti italiani più affermati, Blu. La vera identità di questo artista non è conosciuta e il "Guardian" nel 2011 l'ha 


\section{Ocula ${ }^{\text {Flux_saggi }}$ \\ Occhio semiotico sui media | Semiotic eye on media}

Orlando Paris | Strategie di ridefinizione semantica della città

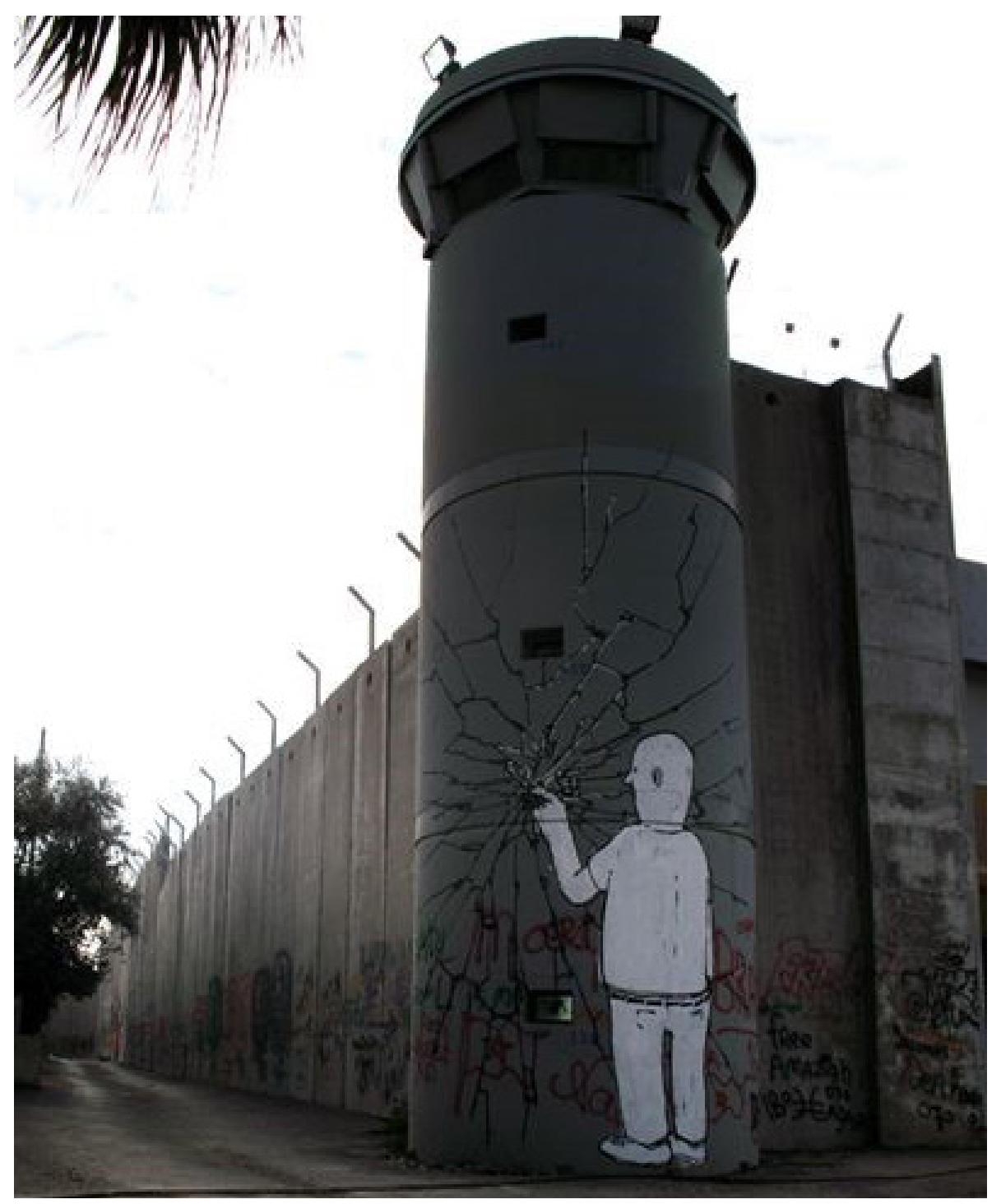

Figura 8. Blu, Cisgiordania

consacrato come uno dei migliori dieci street artist al mondo. Blu interviene sul Muro di separazione nei pressi di Betlemme nel 2007 come si può vedere nella figura che segue (fig. 8).

L'Artista di Senigallia (Bolognese di adozione) ci presenta una vera e propria narrazione: nella rappresentazione un ragazzo (il soggetto protagonista) solamente con la giustapposizione di un dito inizia un processo di distruzione del muro, cioè del supporto grazie al quale l'opera si manifesta. L'opera di Blu è quindi un "segno" che ci mostra qualcosa, in particolare una storia: l'inizio dell'abbattimento della barriera, non così solida visto che basta un dito per iniziare il processo di dissoluzione. Ma allo stesso tempo l'opera si presenta anche come un "meta-segno", come una "riscrittura esplicita" del suo supporto: la barriera/supporto viene fatta emergere e diventa una figura della rappresentazione (tra l'altro rappresenta sé stessa), così facendo viene ri-scritta, 


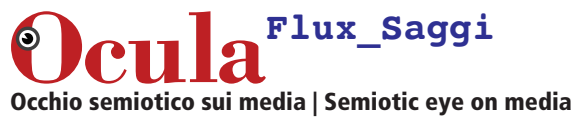

Orlando Paris | Strategie di ridefinizione semantica della città

mostrata come fragile, con delle crepe, nell'atto di dissoluzione. L'osservatore è di fronte a una doppia dinamica di significazione e, quindi, una doppia possibilità di interpretazione: il significato originario del supporto, che è ancora lì riconoscibile e interpretabile con tutte le sue "connotazioni", e la riscrittura artistica, che mostra quel supporto risignificato, debole, incrinato, nell'atto di frammentarsi. Il segno artistico, in questo modo, rende esplicito e visibile il suo congegno "meta-rappresentativo" (funzione riflessiva) e diventa, in altre parole, un segno di una riscrittura, un meta-segno che mostra in modo critico la trasformazione del suo supporto che è un enunciato urbano. È un dialogo polemico quello tra opera e suo supporto, un vero e proprio scontro di "significazioni" che si contrappongono.

Un altro famosissimo artista che ha realizzato diverse opere sul muro in Cisgiordania è Banksy. Questo artista ha realizzato nove opere sul muro di separazione tra Israele e Palestina. Anche in queste occasioni siamo di fronte a segni che mostrano storie che evocano l'abbattimento o il superamento della barriera, ma sono anche meta-segni che si presentano come "ri-scritture esplicite". Nella figura che abbiamo già visto nelle pagine precedenti e che rappresenta un'opera di Banksy (fig. 6), i soggetti protagonisti sono due angioletti impegnati in un programma narrativo volto alla distruzione della barriera. Ma anche in questo caso, come nel precedente (fig. 8), ci troviamo di fronte a un "meta-segno": la rappresentazione ci parla di sé stessa, del proprio supporto. La barriera viene riportata in primo piano e grazie ad un'imperfezione (i moduli con cui la barriera è costruita non sono allineati), che diventa elemento centrale della rappresentazione, viene ri-significata. La figura 9, invece, chiama in causa e coinvolge direttamente l'osservatore che diventa il soggetto protagonista della narrazione rappresentata dall'artista: a questo viene proposto infatti di mettere in atto direttamente il programma narrativo di abbattimento della barriera attraverso il segno di ritagliare lungo la linea tratteggiata. Di nuovo un programma narrativo che propone l'abbattimento della barriera, di nuovo un meta-segno che ci parla di sé stesso, ci parla del suo supporto e lo ri-scrive.

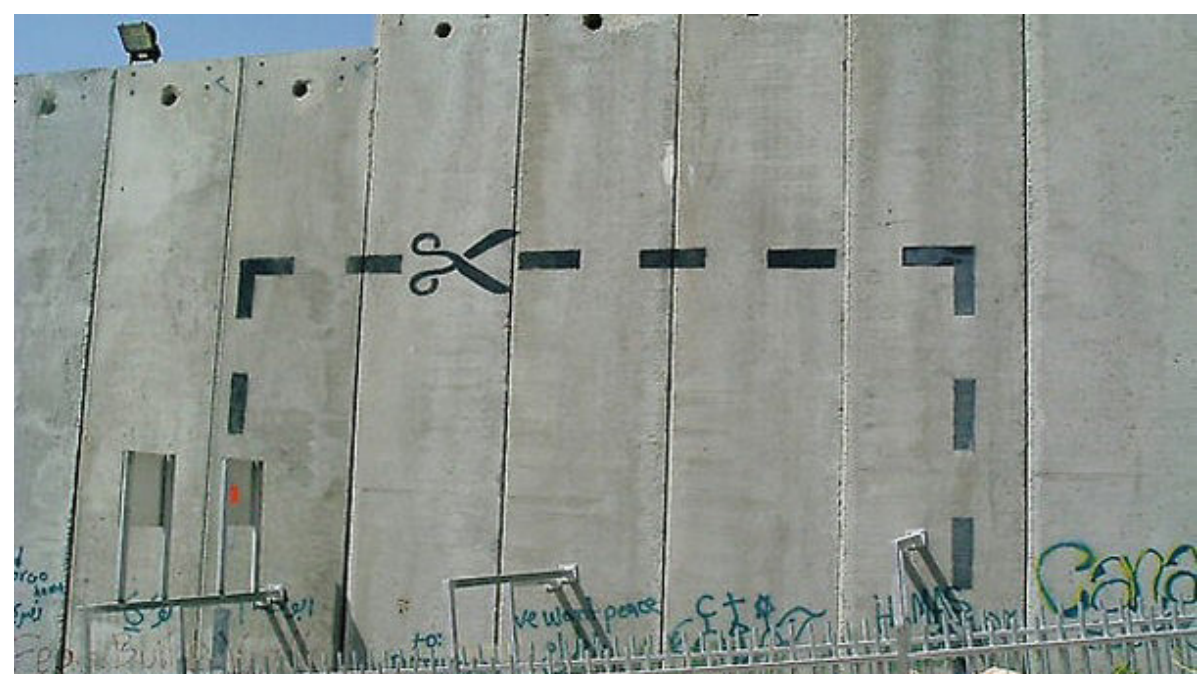

Figura 9, Banksy, Cisgiordania. 


\section{Dcula ${ }^{\text {Flux_saggi }}$ \\ Occhio semiotico sui media | Semiotic eye on media}

Orlando Paris | Strategie di ridefinizione semantica della città

Tutte e nove le opere di Banksy sul muro in Cisgiordania costruiscono, secondo le modalità appena descritte, il rapporto tra rappresentazione artistica $\mathrm{e}$ barriera-supporto. Ma più in generale sono molti gli artisti internazionali che sono intervenuti sul muro e nelle cui opere è rintracciabile questa dinamica, solo per citarne alcuni: l'italiano Filippo Minelli con il suo CTRL+ALT+DELETE, l'artista palestinese Wissam Salsa che realizza una gamba che rompe la barriera, l'artista di Belluno Ericailcane che evoca in diversi modi il superamento del muro anche attraverso il disegno di una scala mobile e tutti gli artisti del collettivo Santa's Ghetto (in tutto 14) che nel 2007 intervengono con una serie di opere che evocano il superamento o l'abbattimento del muro di separazione. Per molte di queste opere, come per le opere citate nelle pagine precedenti (figg. 2-6, 8), si può parlare di meta-segni, rappresentazioni in cui è presente -e in alcuni casi prevale- una dimensione riflessiva e che si presentano prima di tutto come "segni" di ri-scrittura. Sono meta-segni quindi, perché parlano della loro dinamica di funzionamento e ce la mostrano; inoltre ci mostrano la loro "struttura" poiché la mettono al centro della rappresentazione tematizzando il loro "supporto" (la barriera, il muro, il cartello stradale, una cassetta delle lettere, eccetera). Quella che attuano è di fatto una ri-scrittura esplicita poiché ci mostrano il meccanismo che la caratterizza. Nelle prossime righe, invece, mostreremo una dinamica di "riscrittura implicita", la rifunzionalizzazione di un edificio: da fabbrica occupata a museo.

3.3 La storia di un edificio rifunzionalizzato dalla street art: l'ex fabbrica Fiorucci ora Museo dell'Altro e dell'Altrove

Nel marzo 2009 l'ex fabbrica-mattatoio della Fiorucci a Roma, ormai edificio abbandonato, attraverso un'azione dell'organizzazione romana "Blocchi Precari metropolitani" viene occupata per fini abitativi. Nell'occupazione dell'enorme edificio (circa ventimila metri quadrati) sono coinvolte duecento persone, circa sessanta nuclei familiari di diverse nazionalità. Nel 2012 grazie all'antropologo e regista Giorgio De Finis e al film maker Fabrizio Boni nasce il primo progetto artistico legato a questo spazio occupato, la realizzazione del documentario "Space Metropoliz". Proprio grazie al documentario vengono coinvolti anche i primi artisti: Lucamaleonte, giovane street artist romano che disegna la sua opera nell'ampia cucina dell'edificio, e Hogre anche lui artista romano che lavora sui manifesti pubblicitari sovvertendone i significati. Il documentario porta in giro per il mondo la storia di questa occupazione e avvia un circolo virtuoso: gli street artist intervengono sempre più numerosi, le loro opere arricchiscono le pareti della ex fabbrica e, in questo modo, da "Spece metropoliz" (questo era il nome che gli occupanti avevano dato a questo spazio) questa si trasforma nel MAAM (Museo dell'Altro e dell'Altrove). Oggi, grazie agli interventi di affermati street artist provenienti da tutto il mondo, le opere sono diventate più di cinquecento e la ex fabbrica, di fatto rifunzionalizzata, è uno degli spazi d'arte più vivaci della Capitale. Le opere d'arte, quindi, riscrivono e rifunzionalizzano uno spazio e così facendo si ergono a difensori degli occupanti: l'obiettivo è di fatto quello trasformare il supporto (la fabbrica occupata), di riscriverlo come museo e, in questo modo, di garantire il 


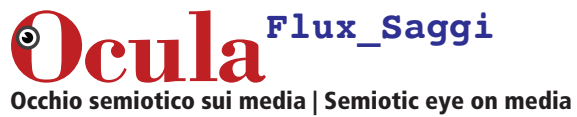

Orlando Paris | Strategie di ridefinizione semantica della città

diritto all'abitare degli occupanti. A tal proposito sono interessanti le parole dell'antropologo Giorgio de Finis, uno degli artefici del MAAM: «Oggi il maam conduce la sua battaglia per assicurare, e non solo immaginare, un futuro migliore agli abitanti di Metropoliz, ancora sotto la minaccia dello sgombero coatto[...]»(De Finis 2017: 16).

Tra gli obiettivi che il MAAM si dà sin da principio possiamo indicare:

1) quello di creare una barricata d'arte a difesa dell'occupazione e dei suoi abitanti (le opere attaccate ai muri e alle strutture della fabbrica sono un esercito schierato); la cacciata di 200 persone (di cui settanta minori) dalle loro case sarebbe comunicato con un trafiletto in cronaca dal titolo: "Bonificata l'area della ex Fiorucci”, mentre la distruzione di cinquecento opere d'arte equiparerebbe chi invia le ruspe ai talebani che cannoneggiano i Buddha in Afghanistan;

2) quello di evitare, o ridurre, l'effetto enclave, rischio che Metropoliz corre dovendo proteggersi dietro un cancello chiuso; il potere attrattivo della collezione del maam e delle sue iniziative periodiche - le aperture del sabato e le inaugurazioni collettive in occasione di solstizi ed equinozi - crea un flusso ininterrotto di visitatori, connettendo di fatto la città meticcia con il resto della Capitale: il maam opera come un dispositivo di incontro e mette in atto precise "strategie" affinché questo sia possibile e fecondo di arricchimento reciproco;

3) quello di proporre e sperimentare un "altro" modello di museo, un "museo abitato" e contaminato dalla vita, che Cesare Pietroiusti ha definito museo reale (vs museo irreale) proprio in una lectio marginalis tenutasi al maam; come pure, di converso, 4) una modalità abitativa informata dalla presenza diffusa e onnipervasiva dell'arte.

Infine, 5) quello di realizzare un'opera corale (una "barriera corallina", come suggerisce Pablo Echaurren) inneggiante al valore della (bio)diversità (il maam non è solo un progetto artistico; ma in quanto super-oggetto è anche un'opera d'arte e in più di una occasione si è anche presentato come un soggetto artistico) (De Finis 2017: 13).

Si tratta di una "ri-scrittura implicita" quella attuata dalla street art nella ex-fabbrica Fiorucci, le opere non prendono in causa direttamente il loro supporto (muro/ex-fabbrica), ma comunque lo riscrivo e lo rifunzionalizzano secondo la dinamica che abbiamo descritto nelle pagine precedenti analizzando i "segni di riscrittura implicita urbana". La cosa più interessante nella storia del MAAM è che questa forza di "ri-scrittura" della street art viene consapevolmente utilizzata per opporsi tatticamente alle strategie istituzionali/politiche di definizione del testo urbano.

\section{Conclusioni: enunciazioni illegittime ed enunciati sovversivi}

In un numero di Ocula curato da Cinzia Bianchi e Silvia Viti (2017), dedicato alla street art e alle dinamiche di musealizzazione che la coinvolgono, Francesco Mazzucchelli (2017) sottolinea come questa pratica artistica modifichi il paesaggio urbano e "l'organizzazione razionale della città pianificata dall'alto, attraverso tattiche di riscrittura e riappropriazione" (Mazzucchelli 2017: 27). Una riscrittura che prevede una particolare forma di enunciazione: 


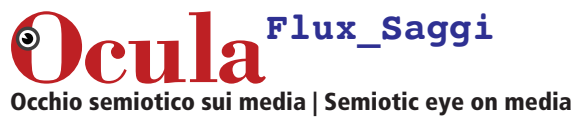

Orlando Paris | Strategie di ridefinizione semantica della città

Un'enunciazione che mette in moto forme di iscrizione di soggettività in spazi che non prevedono, in origine, una voce per tali soggettività e dunque un modo di iscrivere quei "punti di vista di mondi privi di accesso legittimo alla parola in pubblico" (Dal Lago, Giordano 2016: 34). Una pratica, dunque, che si configura in primo luogo come azione sovversiva, illegale, di "segnatura" di un luogo, di "territorializzazione", di messa in scena di identità politiche $\mathrm{o}$ sociali di contestazione o comunque in competizione con quelle egemoniche. (Mazzucchelli 2017: 27)

Una riscrittura, quella della street art, fondata su un atto enunciativo "illegittimo", poiché non previsto nella pianificazione pubblica della città e di conseguenza non ufficiale, non "istituzonale", non legale. Questa enunciazione illegittima produce segni "sovversivi": segni che "sovvertono" e "riscrivono" enunciati urbani che hanno significati e funzioni originariamente "pianificati dall'alto". Questa forza "riscrittrice", che in termini enunciativi corrisponde al ritaglirsi illegale di uno spazio d'espressione lì dove non è previsto, a livello dell'enunciato è rintracciabile proprio nella relazione complessa tra street art e suo supporto che abbiamo provato ad analizzare in questo lavoro: una relazione in cui si manifesta il confronto/scontro tra regimi discorsivi differenti, quello istituzionale e quello delle varie "soggettività" sociali e politiche che abitano la città.

\section{Bibliografia}

Bianchi, Cinzia; Viti, Silvia

2017 "Introduction. Street art: Iconoclasm and Istitutionalization", Ocula, vol.18, n.18, pp. 1-6.

Ferrara, Lamberto; Mondino, Marco; Stano, Simona

2012 "I graffi della protesta", Lexia 13-14, pp. 161-205.

Lancioni, Tarcisio

2013 "Immagini in prospettiva. Forme e figure dell'enunciazione visiva", in Leone, M. e Pezzini I. (a cura di), Semiotica delle soggettività. Per Omar, Roma, Aracne.

Marin, Louis

2001 Della rappresentazione, Roma, Meltemi.

2012 Opacità della pittura. Sulla Rappresentazione nel Quattrocento, Firenze, La Casa Usher.

Marrone, Gianfranco

2009 "Dieci tesi per uno studio semiotico della città. Appunti, osservazioni, risposte", Versus, 109-11, pp. 11-46.

Marrone, Gianfranco; Pezzini, Isabella (a cura di)

2006 Senso e metropoli, Roma, Meltemi.

2008 I linguaggi della città. Senso e metropoli II, Roma, Meltemi.

Mastroianni, Roberto

2013 "Dal segno metropolitano al muralismo artistico", in Mastroianni, R. (a cura di), Writing the city. Graffitismo, immaginario urbano e street art, Torino, Aracne. 


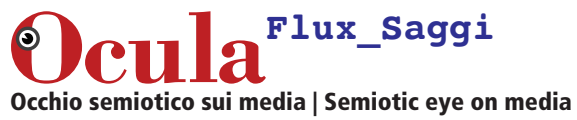

Orlando Paris | Strategie di ridefinizione semantica della città

Mazzucchelli, Francesco

2017 "Street(icono)clashes. Blu vs Genus Bononiae: un caso di iconoclastia urbana", Ocula, vol.18, n.18, pp.22-42.

Mubi Brighenti, Andrea

2008 "The wall and the city", in Lo squaderno, 8, pp.7-9.

2009 "Walled urbs to urban walls - and return? Onthe social life of Walls", in Mubi Brighenti, A. (a cura di), The Wall and the city, Trento, PofessionalDremers.

Paris, Orlando

2019 "Strategie di ridefinizione semantica del muro", Cultura e Comunicazione, 15, pp. 29-34.

Petrilli, Raffaella

2017 "Immaginazione simbolica. Funzioni e significati per la Street art" in Mania, P., Petrilli, R. e Cristallini E. (a cura di), Arte sui muri della città. Street art e Urban art: questioni aperte, Roma, Robin.

Pezzini, Isabella; Finocchi, Riccardo (a cura di)

2020 Dallo spazio alla città. Letture e fondamenti di semiotica urbana, MilanoUdine, Mimesis.

Scalabroni, Luisa

2008 Forme e ruoli della cornice nella rappresentazione pittorica, <http://www. ec-aiss.it/pdf_contributi/scalabroni_20_3_08.pdf>; in rete dal 20/03/2008.

Stano, Simona

2013 "Graffiti e pratiche di ri-scrittura murale", in Mastroianni, R. (a cura di), Writing the city. Graffitismo, immaginario urbano e street art, Torino, Aracne.

Vedovelli, Massimo

2019 "Siena, dall'immagine alla parola e ritorno", Cultura e Comunicazione, 15, pp. 7-12.

Volli, Ugo

2005 «Per una semiotica della città», in id., Laboratorio di semiotica, Roma-Bari, Laterza.

2008 Lezioni di filosofia della comunicazione, Roma-Bari, Laterza.

2009 "Il testo della città- Problemi metodologici e storici", Lexia, 1-2, pp. 9-21.

2013 «Per una semiotica dei graffiti?», in Mastroianni, R. (a cura di), Writing the city. Graffitismo, immaginario urbano e street art, Torino, Aracne.

Orlando Paris è assegnista di ricerca in Semiotica e Filosofia del linguaggio presso l'Università per Stranieri di Siena. Ha conseguito il Dottorato di ricerca presso l'Istituto Italiano di Scienze Umane e Sociali, Scuola Normale Superiore. Tra le sue pubblicazioni ci sono articoli scientifici e saggi di semiotica tra i quali: Il discorso scientifico e la costruzione dell'altro (Pacini Editore, 2017), I discorsi dell'odio. Razzismo e retoriche xenofobe sui social network (con Caterina Ferrini, Carocci, 2019). 
Ocula $a^{\text {Fux_saggi }}$

Occhio semiotico sui media | Semiotic eye on media

Orlando Paris | Strategie di ridefinizione semantica della città 
Ocula $a^{\text {Fux_saggi }}$

Occhio semiotico sui media | Semiotic eye on media

Orlando Paris | Strategie di ridefinizione semantica della città 